\title{
Simulation of Pedestrian Dynamics with Macroscopic and Microscopic Mathematical Models
}

\begin{abstract}
G. Bärwolff ${ }^{1, *}$, M. Chen ${ }^{2}$, H. Schwandt ${ }^{3}$
Here, we collect two parts of a research project on the pedestrian flow modeling. Rapid growth in the volume of public transport and the need for its reasonable, efficient planning have made the description and modeling of transport and pedestrian behaviors as important research topics in the past twenty years. First, we present a macroscopic model for the pedestrian flow based on continuum mechanical balances. Second, we present a new microscopic modelling method to describe the interaction among pedestrians in conflicting situations. A local navigation based on a continuous density estimator is adopted for the configuration of pedestrians' temporary route choices on the tactical level. On the operational level, a balancing mechanism is installed to ensure correct execution of the planned position transitions of the pedestrians. A comparison of the test results of our simulation with a real-world video clip is provided.
\end{abstract}

Keywords: Pedestrian flow, Macroscopic/microscopic models, Pedestrian density and flow measurement, Human crowd experiments.

Manuscript was received on 05/08/2017, revised on 09/11/2017 and accepted for publication on 20/11/2017.

\section{Introduction}

In a close cooperation with engineers we investigate the mathematical description of pedestrian movement by

1. data generation with real world experiments for model validation and parameter calibration,

2. development of

(a) microscopic mathematical models,

(b) macroscopic mathematical models.

We managed the experiments with about 300 students of the TU Berlin and recorded the different constellations of pedestrian streams by a multi-trace recorder as base for generation of individual tracks and a density estimation.

As microscopic models we discuss grid-based approaches rooted in cellular automata and a second ansatz with a combination of force-based and graph-based approach. The microscopic models are discussed in detail, for example, in Chen et al. [3] and Lämmel and Plaue [14].

\footnotetext{
${ }^{*}$ Corresponding Author.

${ }^{1}$ Department of Mathematics, Technical University of Berlin, Berlin, Germany, Email: baerwolf@math.tuberlin.de.

${ }^{2}$ Department of Mathematics, Technical University of Berlin, Berlin, Germany, Email: minjie.chen@ math.tuberlin.de.

${ }^{3}$ Department of Mathematics, Technical University of Berlin, Berlin, Germany, Email: schwandt @ math.tuberlin.de.
} 
Here, we are focused on macroscopic models based on a set of pedestrian-specific coupled partial differential equations. The first discussed model is based on the mass balance and the considered quantities are the pedestrian density and fluxes which give information about the velocities of pedestrian groups.

\section{A Compressible Macroscopic Model}

This macroscopic approach is based on a set of pedestrian-specific coupled partial differential equations. The equations are not derived from the Euler-/Navier-Stokes equations known from fluid and gas dynamics. The specific situation of multi-destination pedestrian crowds with crossing streams requires the development of appropriately adapted methods. This has been targeted by the use of simple heuristics.

Typical applications of these approaches include real-world scenarios like airports, shopping malls, middle to large size buildings, etc., where the participants (i.e., the pedestrians) do not exhibit an overall unanimity and (may) have different and multiple destinations.

Beyond the modeling of the above-mentioned problems, a particular aim of this project will be the development, implementation and test of appropriate computer-based simulation models.

\subsection{The Transport Equation}

Perceiving pedestrian flows as a transport problem, we start with the governing equation that describes the mass flow as

$$
\frac{\partial \rho_{i}}{\partial \vartheta}+\nabla \cdot\left(\rho_{i} v_{i}\right)=0
$$

where $\vartheta$ denotes the time, and $i \in\{1, \ldots, n\}$ with $n$ being the number of pedestrian "types" or "species" distinguished by certain properties. The desired velocity would be a frequent example of such a property. Furthermore, $\rho_{i}$ is the current density and $v_{i}$ is the current velocity of a species in a given computational domain.

Since pedestrian dynamics cannot be entirely described as a physical phenomenon, the parts of the equation can do well with some discussions.

\subsection{Measuring Pedestrian Density}

In physics, mass density $\rho$ has units $[\rho]=\frac{\text { mass }}{\text { volume }}$. However, this does not seem to be the best fit for the problem considered here. The definition $[\rho]=\frac{\text { mass }}{\text { area }}$, used, for example, in Moussaïd et al. [15], similarly includes "mass" which the authors needed to model inertial effects, but are not included in our model.

Concerning pedestrian inertial mass, we require the following assumption: 
Due to the smoothness of the controlling fields, we assume that it is not necessary to describe mass (inducing inertial behavior in the model). This way, we assume that the pedestrians may follow (adapt speed and heading) to the controlling fields without significant lag by means of internal impetus, decision and physical strength.

Therefore, one natural way to measure the pedestrian density in this setting is to use $[\rho]=$ $\frac{\text { pedestrians }}{\text { area }}$, which implicitly relies on a certain amount of homogeneity of the pedestrian crowd in the considered sample. An even smarter approach is presented in Predtechenskii and Milinskii [17] by defining the area that a specific pedestrian occupies. This area depends on, for example, whether the pedestrian is a child, an adolescent or an adult, which cloths the pedestrian wears (summer or winter cloths), how much luggage the pedestrian carries and so on. This yields an appropriate dimensionless measure, $[\rho]=\frac{\text { pedestrians' needed area }}{\text { availabel area }}$. Since the area occupied by a pedestrian is not always readily available as input, we choose the former definition of density as pedestrians per area.

In our model, we use normalized densities: $\rho_{i}, \rho \in[0,1]$ with $\rho=\sum_{i=1}^{n} \rho_{i}$. A value of $\rho=1$ would for instance mean $5.4 \frac{\text { pedestrians }}{\text { area }}$, according to Weidmann [22], and up to $10 \frac{\text { pedestrians }}{\text { area }}$, according to other sources (see, e.g., Schadschneider et al. [21] for a discussion).

\subsection{Transport Velocity}

The primary goal is to find a sensible functional relation $v_{i}=v_{i}\left(\rho_{1}, \ldots, \rho_{n}\right)$ that yields a nonlinear system for realistic cases.

In the literature, one frequently discriminates between a planned (e.g., "external" in Cristiani et al. [5] or "tactical" in Schadschneider et al. [21]) and an instantaneous (e.g., "intelligent" in Cristiani et al. [5] or "operational" in Schadschneider et al. [21]) velocity. In our opinion, this differentiation makes sense in the context of categorizing the cause of an action taken by a pedestrian.

Our approach is slightly more pragmatic and accounts for three different types of decisions. Pedestrians

(1) choose a direction they wish to go,

(2) choose a speed for walking in the chosen direction based on local conditions,

(3) alter speed and walking direction in order to locally avoid densely populated areas (prefer the direction of $-\nabla \rho$ ).

Therefore, we decompose the velocity as follows:

$$
v_{i}=a_{i} V d_{i}^{\mathrm{i}}-b_{i} W d^{\mathrm{l}}
$$

where

$V \in[0,1]$ is a normalized speed determined by a fundamental diagram (see Section 2.6),

$d_{i}^{\mathrm{i}}$ is a unit vector field pointing into the direction of the desired heading, 
$d^{\mathrm{l}}$ is a directional vector field for local correction (not necessarily of unit length; see below) (since it depends on the total density $\rho$, it is common to all pedestrian species),

$a_{i}$ and $b_{i}$ are constants: $a_{i}$ stands for the absolute value of the wished velocity and $b_{i}$ is a measure for avoiding regions of high density,

$W=1-V$ reflects the operational shift from wanting to reach the desired target to reacting to local encounters with other pedestrians at high densities.

Summarizing this, the term $a_{i} V d_{i}^{\mathrm{i}}$ stands for the gradient driven part of the velocity and $b_{i} W d^{\mathrm{l}}$ decries the influence of high density regions on the velocity of pedestrians.

A model for two pedestrian species with just the $a_{i} V d_{i}^{i}$ term present has been investigated in Berres et al. [1] with a focus on discussing the mathematical foundation. This investigation highlights some shortcomings of the model with respect to the simulation of real-world scenarios. The authors suggest to introduce (cross) diffusion terms in order to solve these problems. Since the meaning of these terms in the context of real-life applications seems to be obscure, here we prefer to introduce the $b_{i} W d^{\mathrm{l}}$ term.

\subsection{Desired Heading}

The term $d_{i}^{\mathrm{i}}$ describes the pedestrian's choice of a walking direction, and is based on spatial information from the vicinity and the global environment of the pedestrian:

$$
\begin{aligned}
& \Delta \phi_{j}^{(i)}(\vartheta)=r_{j}^{(i)}(\vartheta), \\
& \phi_{i}(\vartheta)=\sum_{j} \phi_{j}^{(i)}(\vartheta), \\
& d_{i}^{j}(\vartheta)= \begin{cases}\frac{\nabla \phi_{i}(\vartheta)}{\left|\nabla \phi_{i}(\vartheta)\right|}, & \text { if }\left|\nabla \phi_{i}(\vartheta)\right| \neq 0, \\
\text { random unit vector, } & \text { if }\left|\nabla \phi_{i}(\vartheta)\right|=0 .\end{cases}
\end{aligned}
$$

With the formula (4) we add the different potentials coming from global influences, like the shape of the considered domain or local influences, like the local density.

The subscript $j$ of $\phi_{j}^{(i)}(\vartheta)$ denotes the influence type (for example, global, local, etc.), and the superscript $i$ denotes the considered species.

Therefore, according to the assumption of continuous influences, $d_{i}^{j}$ is based on source and boundary terms of $j$ (partially) solved Poisson equations for each pedestrian species $i$ (reflecting $j$ different influencing factors). The $\alpha_{j}^{(i)}$ are constant weights, and the $f_{j}^{(i)}(\vartheta)$ are source terms derived from spatially distributed information - for example, the density $\rho_{k}(\vartheta)$ of some pedestrian species. This kind of flow, the direction of which is derived from a potential, has been investigated in Hughes [9].

The parameters in the above equations have to be chosen very specifically, and finding appropriate settings is an open task for the application of this model. 
With a special choice of the right hand side of (3), we can model global or local influences by potentials. A detailed discussion of this point is given in Huth et al. [10].

\subsection{Introducing the Gradient Term}

There are a number of possible approaches to introduce a gradient term. Notable are the following two:

$$
\begin{aligned}
& d^{\mathrm{l}(\vartheta)}= \begin{cases}\frac{\nabla \rho(\vartheta, x)}{|\nabla \rho(\vartheta, x)|}, & \text { if }|\nabla \rho(\vartheta, x)|>0, \\
0, & \text { if }|\nabla \rho(\vartheta, x)|=0,\end{cases} \\
& d^{\mathrm{l}(\vartheta)}= \begin{cases}\frac{\nabla \rho(\vartheta, x)}{|\nabla \rho(\vartheta, x)|}, & \text { if }|\nabla \rho(\vartheta, x)|>1, \\
\nabla \rho(\vartheta, x), & \text { otherwise. }\end{cases}
\end{aligned}
$$

Concerning (5), it has to be noted that $d^{\mathrm{l}}(\vartheta, x)$ is not necessarily continuous with respect to $x$ at points, where $|\nabla \rho(\vartheta, x)|=0$. Another problem obviously present with this term is that it may show large scatter where the density is high. This can lead to the violation of the condition $\rho_{i}, \rho \in[0,1]$, because of numerical overshooting. Another risk is the numerical oscillation of the solution (which might be interpreted as remaining erratic pedestrian activity at high densities in certain situations). This term might be viewed as carrying some random disturbances as discussed in Helbing et al. [7] or Radzihovsky and Clark [18], which produces less effective motion (stronger clogging tendency due to the "freezing by heating effect", discussed there). The measurement of the mobility there did not show an increase of flux with more "thermal" motion at all. This is due to the inhibition of lane formation because the effect of lane formation yields an enhancement of flux. The observed "freezing by heating effect" has been considered for modeling panic situations, where it might well make sense. However, such scenarios are beyond the scope of this paper.

The gradient term defined by (6) is more likely to be the rule applied by pedestrians under normal conditions, because it is more efficient than the term given by (5).

Another argument in favor for (6) is given by the fact that the key idea of the macroscopic approach is to average the behavior of several pedestrians, and thus to smooth out random disturbances caused by single pedestrians at sufficiently large scales.

\subsection{Walking Speed and Fundamental Diagram}

A uni-directional flux can be defined by $J=\rho_{i} V\left(\rho_{1}, \ldots, \rho_{n}\right) d_{i}$. For the case of $J=\rho V(\rho) d$, the three quantities are related by a fundamental diagram. Fundamental diagrams have been determined by a number of authors, with a relatively wide range of different results that cannot be used to deduce a general law. According to Schadschneider et al. [21], the values found in the literature for the maximum pedestrian density, where movement is possible at all, vary from $3.8 / \mathrm{m}^{2}$ to $10 / \mathrm{m}^{2}$. Another controversially discussed issue is how $V$ depends on whether movement is uni-directional or multidirectional. For more details, see Huth et al. [11] and [12]. 
Despite the issues described above, we have evaluated the impact of different fundamental diagrams on quantitative and qualitative properties of the solutions. The differences are large enough to indicate the need for a better approximation in this respect. The fundamental diagrams we have tested are

$$
\begin{aligned}
& V(\rho)=1-\rho, V(\rho)=(1-\rho)^{2}, V(\rho)=1-\rho^{2}, \\
& V(\rho)=1-\exp (-1.913 / 5.4(1 / \rho-1)) .
\end{aligned}
$$

Note that, compared to Weidmann [22, p. 65], (7) employs the normalization conditions $V \in[0,1]$ and $\rho \in[0,1]$.

\subsection{Simulation Example $-90^{\circ}$ Encounter}

The above discussed boundary value problem completed by appropriate boundary conditions (see Huth et al. [10]) is solved using the Finite-Volume package OpenFOAM. Fig. 1 shows the results of two pedestrian streams crossing with an angle of $90^{\circ}$. A formation of dynamically reconfiguring clusters can be observed.
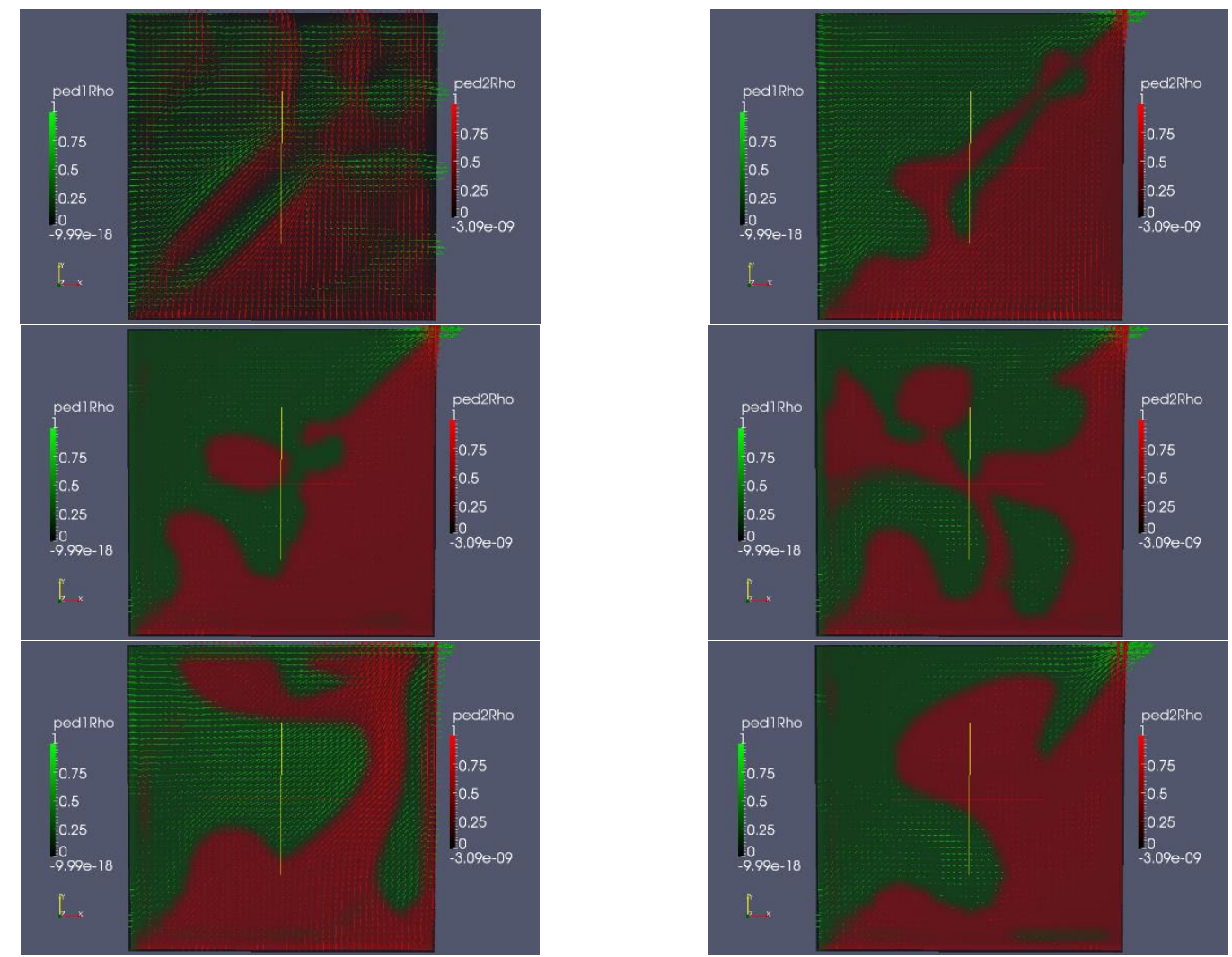

Figure 1. Time steps 5, 10, 20, 40, 60 and 80 of the simulation of a $90^{\circ}$ encounter of two species. Shown are density and flux of one species coming from left. The length of the arrows indicates flux strength, the grade of darkness indicates the density of the species. The crossing species coming from bottom is located in the light gray or white regions of the area. 


\section{Microscopic Modelling}

Pedestrian dynamics as an interdisciplinary research field studies the human social behavior from a mathematical (or engineering) perspective. In the microscopic category, the social force model Helbing et al. [6], defined in a continuous space, has been a forerunner. In this model, the interaction among the pedestrians is considered to be the result of physical forces in the context of human social behavior. The social force model, when applied to describe the local interaction among the pedestrians, can be combined with further simulation components such as cognitive modelling, proposed by Moussaïd et al. [15]. In the same microscopic category, cellular automata (CA) models were presented by Burstedde et al. [2], Keßel et al. [13], Schadschneider [19] and their extensions were established on discrete geometric structures, to be more exact, on homogeneous grids. The state evolution of the grid cells can thus be used to describe the system dynamics respecting the pedestrians.

A differentiation of the pedestrian behavior has been elaborated in Hoogendorn and Bovy [8]. At the highest level, which is called the strategic level, pedestrians' activity patterns concerning concrete route planning and signals indicating departure and arrival are studied. On the tactical level, activity scheduling and temporary route choice become the concern. On the operational level, walking behavior of the pedestrians as the result of local interaction among them are studied. Incidents on the strategic level are considered as a priori knowledge in the current text. Our study will concentrate on the intermediate level and the lowest level.

\subsection{Operational Level}

Our approach is defined on a square grid similar to the geometric structures in the CA models. In discrete form, position transition can be thus written as $(\mathrm{d} x, \mathrm{~d} y)$ with $\mathrm{d} x, \mathrm{~d} y \in \mathbb{Z}$, measured in the grid cell length. In case $\mathrm{d} x, \mathrm{~d} y \in\{-1,0,1\}$, the position transition is referred to as an elementary step. The pedestrians to be simulated are sometimes called particles.

Most existing CA models assume a maximum discrete speed of $v_{\max }=1$, that is, in a simulation cycle, only elementary position transitions (sometimes the diagonal moves $\mathrm{d} x, \mathrm{~d} y \in\{-1,1\}$ are excluded in addition). CA models construct, dynamically for each particle, a so-called transition matrix which indicates the particle's preference of the elementary steps at the current moment:

$$
P=\left(\begin{array}{lll}
p_{-,+} & p_{0,+} & p_{+,+} \\
p_{-, 0} & p_{0,0} & p_{+, 0} \\
p_{-,-} & p_{0,-} & p_{+,-}
\end{array}\right)
$$

with subscripts of $p$ addressing the possible elementary steps, where 1 and -1 are shortened to signs. The transition matrix items are to be normalized to sum up to 1 . In case of a potential conflict with other particles, a solution will be sought by weighing the corresponding transition matrix items. An underlying disadvantage of this construction is that, when a particle has more possible elementary step choices, its transition matrix items will have lower weights, consequently in competition with others - these may or may not have many possible elementary step choices - targeting a specific destination grid cell position, it will have a smaller chance to be favoured in the conflict resolution. Despite the similar geometric structure, our grid-based approach is completely different. On the operational level, position transitions of the particles in a simulation cycle will be decomposed into elementary steps of the individual particles. This decomposition should be carried out accurately, 
whenever possible. While elementary step execution of the particles is reciprocal, the elementary step choices should be performed in a balanced manner for all the particles in a simulation cycle.

\subsection{Balancing Elementary Steps}

Our previous work Chen et al. [3] offered a general solution for $v_{\max }>1$. Respecting an arbitrary position transition $(\mathrm{d} x, \mathrm{~d} y)$, the number of the elementary steps needed to realize it will be called its topological length. We request that, in the ideal case, topological length and geometric length of an arbitrary position transition should be equal.

Without loss of generality, we consider the case $\mathrm{d} x, \mathrm{~d} y>0$. On the operational level, the possible elementary steps can be confined to one step in grid cell in the $x$-direction, one step in the $y$-direction and one diagonal step in both directions. We assign probability numbers $p_{x}, p_{y}, p_{x y} \in[0,1]$ to these elementary steps which sum up to 1 :

$$
p_{x}+p_{y}+p_{x y}=1
$$

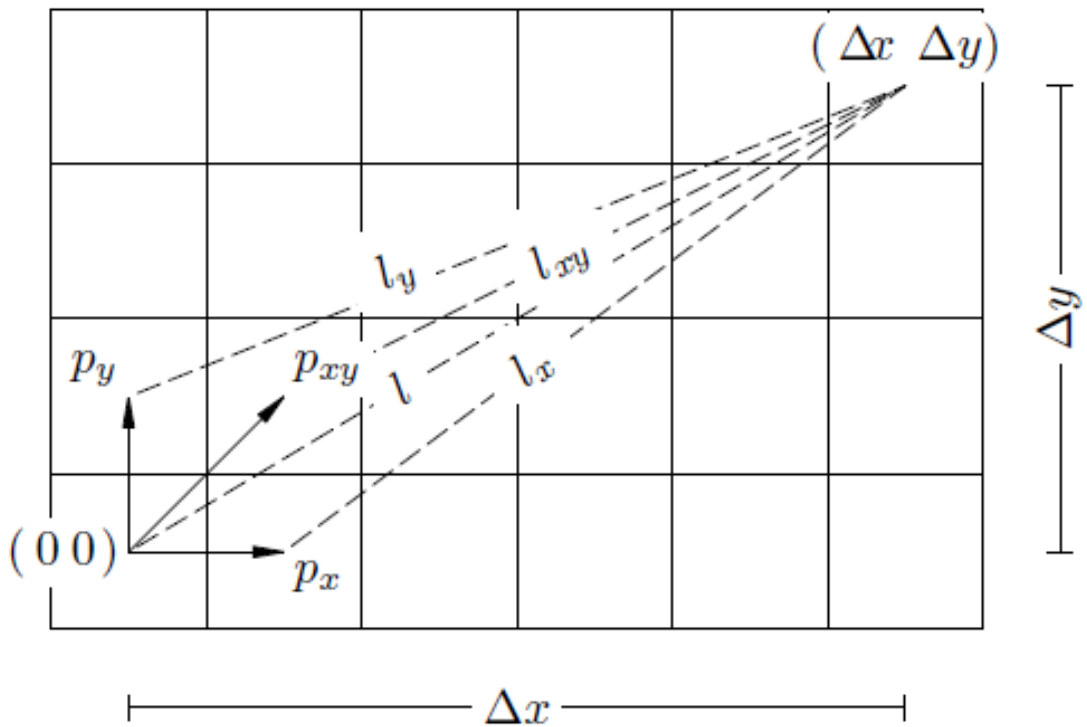

Figure 2. Neutralization of the topological length of a diagonal elementary step. Current elementary steps are associated with probabilities $p_{x}, p_{y}$ and $p_{x y}$, respectively.

Imposing the equality of topological length and geometric length, we have

$$
p_{x}\left(1+l_{x}\right)+p_{y}\left(1+l_{y}\right)+p_{x y}\left(1+l_{x y}\right)=l,
$$

in which the geometric lengths of the current position transition and the rest position transitions after a corresponding elementary position transition will be written as

$$
\begin{aligned}
& l=\sqrt{(\mathrm{d} x)^{2}+(\mathrm{d} y)^{2}}, \\
& l_{x}=\sqrt{(\mathrm{d} x-1)^{2}+(\mathrm{d} y)^{2}}, \\
& l_{y}=\sqrt{(\mathrm{d} x)^{2}+(\mathrm{d} y-1)^{2}},
\end{aligned}
$$




$$
l_{x y}=\sqrt{(\mathrm{d} x-1)^{2}+(\mathrm{d} y-1)^{2}}
$$

see Fig. 2. In addition, we request that the likelihood to carry out an axis-parallel elementary step should correlate with the geometric lengths of the components $\mathrm{d} x$ and $\mathrm{d} y$, and in this sense, we have the next bounding condition:

$$
p_{y} \cdot \mathrm{d} x=p_{x} \cdot \mathrm{d} y
$$

Solving (8) - (10), we have

$$
\begin{aligned}
& p_{x}=\frac{l-l_{x y}-1}{l_{x}+\frac{\Delta y}{\Delta x} l_{y}-\left(1+\frac{\Delta y}{\Delta x}\right) l_{x y}}, \\
& p_{y}=\frac{\Delta y}{\Delta x} p_{x} \\
& p_{x y}=1-p_{x}-p_{y} .
\end{aligned}
$$

An elementary step can be performed accordingly. It is to be noted that an elementary step choice may or may not be performed successfully, depending on the grid cell occupancy by the other particles in the system geometry.

\subsection{Balancing Collection Position Transitions}

To perform the elementary step choices for all the particles on a balanced basis, the simulation cycle will be divided into $m$ equal intervals $\left(m \geq v_{\max }\right)$. An individual particle at a current discrete speed $v\left(0 \leq v \leq v_{\max }, v_{\max }\right.$ and $v$ not necessarily integers $)$ is expected to perform $v$ successful elementary steps by which the planned position transition in the simulation cycle with a geometric length of $v$ can be realized. Chen et al. [4] proposed the following procedure.

\section{procedure OPERATE:}

parameter: a collection of particles;

mark all particles as "unprocessed";

initialize local variable for each particle $a \Leftarrow 0$;

$i \Leftarrow m$;

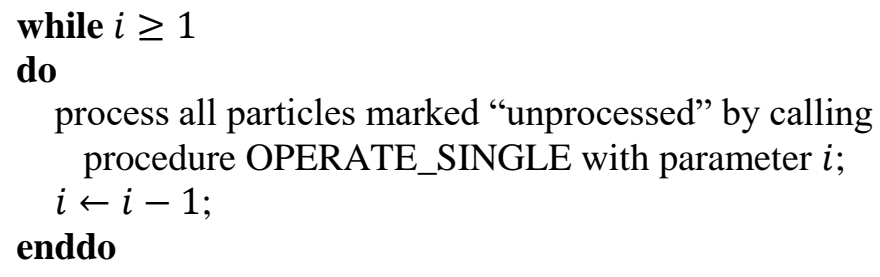

The next procedure processes the individual particles separately. 


\section{procedure OPERATE_SINGLE:}

parameter: particle (each with a local variable $a$ ), $i$;

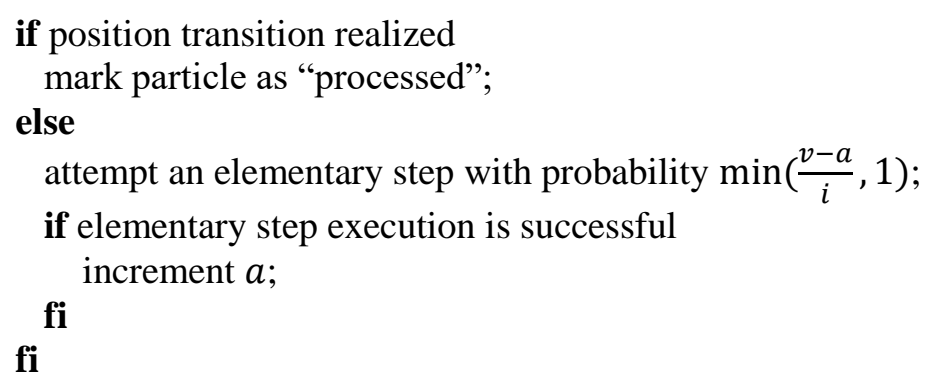

\section{return}

Here, the parameter $i$ serves as a time stamp. We see that an unsuccessful elementary step in the current simulation cycle would lead to a higher probability for a reattempt in the next time interval in the simulation cycle. Consequently, this yields a solution for the conflicts among the particles on the operational level. The position transitions of the particles will be realized as much as possible. Conflict resolution on the intermediate level will be detailed in the following text.

\subsection{Tactical Level}

On the tactical level, temporary route choices targeting new grid cell positions will be prepared for the particles in every simulation cycle.

\subsubsection{Continuous Density}

We will propose a navigation method for the particles to choose their temporary destinations for the next simulation cycle. The method is based on continuous density estimation. First, we go over the notion of density estimation.

In an observation area $\Omega \in \mathbb{R}^{2}$ populated with a collection of particles, the global density can be estimated by counting the number of the particles. In analogy, the local density respecting a given neighborhood can be calculated. However, discrete local density calculated in such a way has poor smoothness.

Let $d_{i}$ denote the distance from particle with label $i$ to its nearest neighboring object (this can be a physical obstacle or another particle) in the whole observation area. The position of this particle will be written as $\mathrm{x}_{i}$. Let $I$ denote the set of the particle labels. Our previous work Plaue et al. [16] suggested a continuous density estimator induced by a collection of particles,

$$
\rho_{I}(\mathrm{x})=\frac{1}{\pi} \sum_{i \in I} \frac{1}{\left(\lambda d_{i}\right)^{2}} \cdot e^{-\frac{\left|\mathrm{x}_{i}-\mathrm{x}\right|_{2}^{2}}{\left(\lambda d_{i}\right)^{2}}},
$$

with an additional smoothing parameter $\lambda(\lambda>0)$. An advantage of (11) is that, with $d_{i} \rightarrow 0$ (that is, the particle crowd becomes infinitesimally dense), it can be approximated by the discrete local density at position $\mathrm{x}$ (consult the original text for the proof). 
On our square grid, we write cell position $\mathrm{x}=(x, y)$ in discrete form. In particular, particle position has the discrete form $\mathrm{x}_{i}=\left(x_{i}, y_{i}\right)$.Accordingly, distances will be converted into discrete forms as well. In addition, we set $\lambda=\sqrt{2}$. Now, we apply the definition of continuous density $\rho$ on cell positions in discrete form:

$$
\rho_{I}(x, y)=\frac{1}{\pi} \sum_{i \in I} \frac{1}{2 d_{i}^{2}} \cdot e^{-\frac{\left(x_{i}-x\right)^{2}+\left(y_{i}-y\right)^{2}}{2 d_{i}^{2}}} .
$$

On a fully populated grid, $d_{i}$ becomes 1 for all $i \in I$.

On the other hand, let us consider the sum

$$
S=\sum_{x=-\infty}^{\infty} e^{-\frac{x^{2}}{2}}
$$

Numerical computation shows that the value of $S$ is very close to $\sqrt{2 \pi} .^{4}$ If the fully-populated grid grows to be infinite, we will have

$$
\rho_{I}(x, y)=\frac{S^{2}}{2 \pi}
$$

whereas the right side has a value very close to 1 . This is exactly what one might expect from the discrete local density estimator mentioned above. Hence, the continuous density calculated in discrete form (12) will be adopted. An example is provided in Fig. 3.

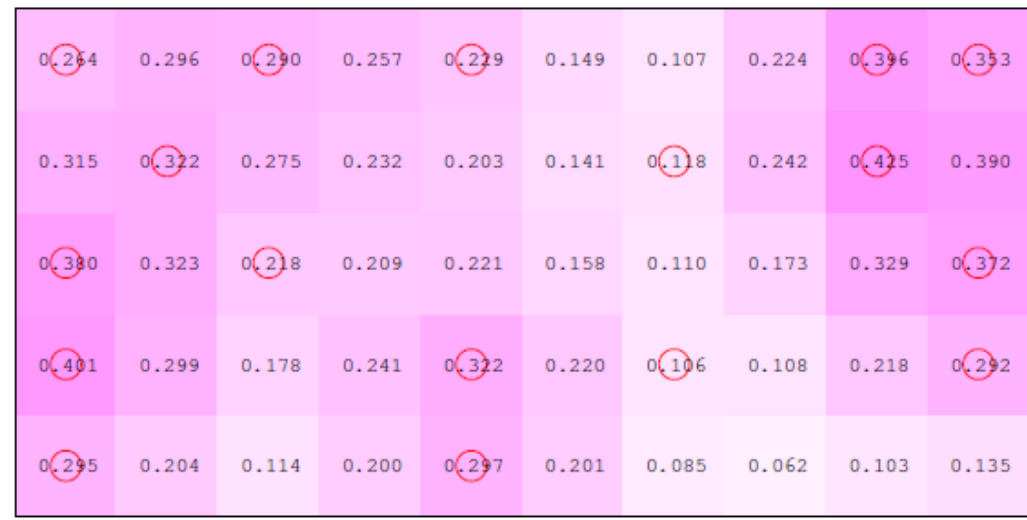

Figure 3. An example of continuous density (expressed in numerical values) in discrete form on a square grid. Particles (shown in circles) belong to the same group. The boundary of the grid is considered as obstacles in the evaluation of $d_{i}$.

The particle label set $I$ can be partitioned into subsets to address different groups of particles. Let $A$ be the label set of a group of particles. The set of the labels of the remaining particles - or, to be more exact, the labels of the particles from all foreign groups - can be written as $\bar{A}$. For a partition of

\footnotetext{
${ }^{4}$ With eight identical significant figures. Mathematically, it is also possible to derive proper upper and lower bounds for $S$.
} 
$I$ by exactly two groups $A$ and $B$, there is simply $\bar{A}=B$. The continuous density in discrete form induced by an arbitrary group $A$ of particles can be written as $\rho_{A}(x, y)$.

\subsubsection{Local Navigation}

It is verified by empirical observations that pedestrians tend to follow their own group members and avoid members of foreign groups. Hence, we define the so-called density overlay as follows:

$$
o_{A}(x, y)=\rho_{\bar{A}}(x, y)-\rho_{A}(x, y)
$$

It is obvious that a grid cell position $(x, y)$ associated with a small density overlay $o_{A}$ will be "attractive" for particles of group $A$. Therefore, on the tactical level, the grid cell position with the lowest density overlay can be chosen as a temporary destination in the current simulation cycle.

We notice in (13) that the attraction incurred by one's own group can be counterbalanced by the foreign groups. This becomes a disadvantage when high densities are involved. We introduce two empirical threshold values for group-induced densities $\theta_{A}$ and $\theta_{\bar{A}}$ and revise (13) as

$$
o_{A}^{\prime}(x, y)= \begin{cases}1, & \text { if } \rho 0_{\bar{A}}(x, y) \geq \theta_{\bar{A}} \\ \rho_{\bar{A}}(x, y)+\rho_{A}(x, y), & \text { if } \rho_{\bar{A}}(x, y) \leq \theta_{\bar{A}} \text { and } \\ & \rho_{\bar{A}}(x, y)>\theta_{A} \\ o_{A}(x, y), & \text { otherwise. }\end{cases}
$$

We take a closer look of (14). In the first case, the induced density by foreign particles is above the corresponding threshold value $\theta_{\bar{A}}(x, y)$, the new overlay value will be 1 , which is, as we recall, the maximum possible density in approximation on the square grid, and thus the current grid cell position $(x, y)$ becomes absolutely unfavorable for all potential incoming particles. If otherwise, as in the second case, the induced density of one's own group $\rho_{A}(x, y)$ is above the corresponding threshold value $\theta_{A}$, the induced density of one's own group will be considered as a negative element and we will negate this (which is, the original negative term in (13) in evaluating the overlay).

Similar to the first case, the current grid cell position will be unfavorable as well. In the third case, when induced density by one's own group and foreign groups are both under the corresponding threshold value, the original definition (13) will be applied. In fact, by setting $\theta_{A}=\theta_{\bar{A}}=1$ (approximated maximum possible density), (13) becomes a special case of (14).

We notice that $o_{A}^{\prime}(x, y)$ is not a continuous function. On the other hand, in modelling pedestrian dynamics, the so-called personal space of the pedestrians must be respected. In other words, although to some extent, pedestrians may be treated as particles, they should not be compressed exceeding a certain limit. In discrete modelling of pedestrian dynamics, in particular in grid-based methods, pedestrians are assigned exclusive spaces. The easiest solution is to define the grid cells with a size resembling that of the exclusive personal space of the pedestrians. A common configuration for the grid cell size in the existing CA models is $0.4 \mathrm{~m}$ by $0.4 \mathrm{~m}$. Pedestrians' influence on others diminishes significantly outside the range of this personal space. Recalling that density can be considered as the inverse of the space, it is not surprising that our density overlay function (14) is not continuous, although the empirical threshold values $\theta_{A}$ and $\theta_{\bar{A}}$ remain to be calibrated. 


\subsection{Simulation Results}

The system dynamics are defined by the collected state evolution of the particles in the simulation system. On the tactical level, the simulation cycle is set to have a time length of one second. The choice of such a time length enables the particles to make or adapt their decisions on the tactical level in a way similar to real pedestrians. Within a simulation cycle, interaction among the particles (pedestrians) is considered to be on the operational level.

Each particle will be given a global walking direction (pointing to an exit, etc.). In each simulation cycle, similar to the cognitive modelling in Section 3, every particle searches for a grid cell position within a perception area defined by a visibility angle $\phi$ from its current position at its default speed; see Fig. 4 for an illustration. A grid position for the next position transition will be selected after comparing the corresponding overlay values. The visibility angle $\phi$ has been defined to be $75^{\circ}$. The size of $\phi$ has been chosen with consideration of real pedestrians' possible changes of their walking directions within a relatively short time span of one second (that is, the length of a simulation cycle) to adapt themselves to the new environment. No further navigation method will be applied.

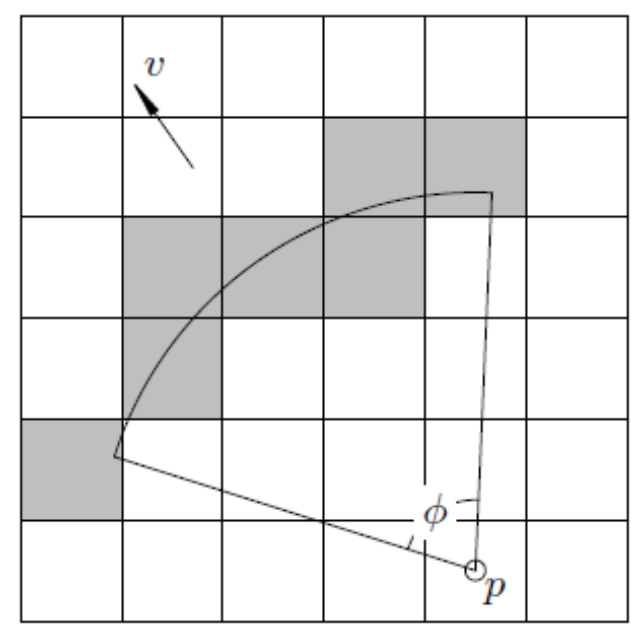

Figure 4. Within its visibility angle $\phi=75^{\circ}$, particle $p$ on the tactical level faces the choice of a proper grid cell position by density overlay

On the operational level, each particle will carry out a series of elementary steps through which its local position transition should be realized. The execution of the elementary steps has already been detailed in Section 3.1 and 3.2. The balancing mechanism ensures all particles equal chances in carrying out their elementary steps in the simulation cycle when local conflicts among them are inevitable.

During the Lange Nacht der Wissenschaften ${ }^{5}$ (German: Long Night of the Sciences) 2010 a couple of experiments were conducted in the Mathematics Department building of Technische Universität Berlin. The experiments were video-recorded to show the interaction among pedestrians in groups. In the experiments, participants were given instructions to walk in a certain direction (toward an exit, etc.).Local position conflicts were resolved by participating pedestrians themselves without guidance or interference from the experiment organizer.

\footnotetext{
${ }^{5}$ Homepage https://www.langenachtderwissenschaften.de.
} 

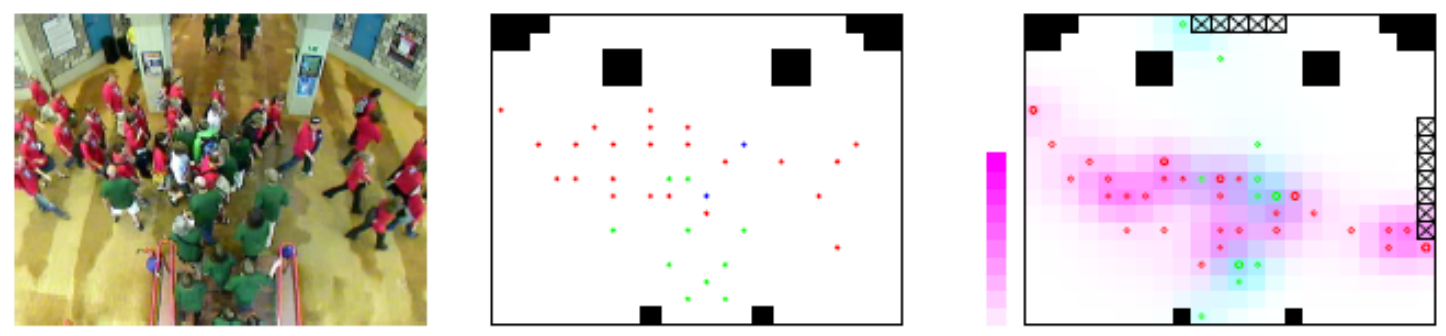

Figure 5. Experiment and the Test Case

\section{Acknowledgement}

The authors gratefully acknowledge the support of Federal Ministry for Economic Affairs and Energy of Germany for the project VP2653402RR1 and federal state of Berlin/Investitionsbank Berlin for the project 10153525 .

\section{References}

[1] Berres, S., Ruiz-Baier, R., Schwandt, H. and Tory, E.M. (2011), An adaptive finite-volume method for a model of two-phase pedestrian flow, Networks and Heterogeneous Media, 6, 401-423.

[2] Burstedde, C., Klauck, K., Schadschneider, A., and Zittartz, J. (2001), Simulation of pedestrian dynamics using a two-dimensional cellular automaton, Physica A, 295, 507-525.

[3] Chen, M.-J., Bärwolff, G., and Schwandt, H. (2010), A study of step calculations in traffic cellular automaton models. In Proceedings of the 13th International IEEE Conference on Intelligent Transportation Systems, IEEE Conference Publications: http://ieeexplore.ieee.org/xpl/mostRecentIssue.jsp?punumber=5610907 , pp. 747-752.

[4] Chen, M.-J., Bärwolff, G., and Schwandt, H. (2014), Simulation of pedestrian dynamics with density control on a regular grid. In Weidmann, U., Kirsch, U., and Schreckenberg, M. (eds.), Pedestrian and Evacuation Dynamics 2012, Springer International Publishing Switzerland, Cham: pp. 949-958.

[5] Cristiani, E., Piccoli, B., and Tosin, A. (2010), Modeling self-organization in pedestrians and animal groups from macroscopic and microscopic viewpoints. In Bellomo, N., Naldi, G., Pareschi, L., Toscani, G. (eds.), Mathematical Modeling of Collective Behavior in Socio- Economic and Life Sciences, Modeling and Simulation in Science, Engineering and Technology, Birkhäuser: Boston, pp. 337-364.

[6] Helbing, D., Farkas, I.J., and Vicsek, T. (2000), Simulating dynamical features of escape panic, Nature, 407, 487-490.

[7] Helbing, D., Farkas, I.J., and Vicsek, T. (2000), Freezing by heating in a driven mesoscopic system, Phys. Rev. Lett., 84(6), 1240-1243.

[8] Hoogendoorn, S.P., and Bovy, P.H.L. (2004), Pedestrian route-choice and activity scheduling theory and models, Transportation Research Part B, 38, 169-190.

[9] Hughes, R.L. (2002), A continuum theory for the flow of pedestrians, Transportation Research Part, B, 36, 507-535.

[10] Huth, F., Bärwolff, G., and Schwandt, H. (2012), A macroscopic multiple species pedestrian flow model based on heuristics implemented with finite volumes. PED 2012 Conference Proceedings. 
[11] Huth, F., Bärwolff, G., and Schwandt, H. (2012), Some fundamental considerations for the application of macroscopic models in the field of pedestrian crowd simulation, TU Berlin Preprint ID 2012/16, http://www.math.tu-berlin.de/menue/forschung/veroeffentlichungen/ preprints-2012.

[12] Huth, F., Bärwolff, G., and Schwandt, H. (2012), Fundamental diagrams and multiple pedestrian streams, TU Berlin Preprint ID 2012/17, http://www.math.tuberlin.de/menue/forschung/veroeffentlichungen/preprints-2012.

[13] Keßel, A., Klüpfel, H., Wahle, J., and Schreckenberg, M., Microscopic simulation of pedestrian crowd motion, In [20], pp. 193-200. ISBN 978-3-540-42690-5.

[14] Lämmel, G., and Plaue, M. (2014), Getting out of the way: collision avoiding pedestrian models, Proceedings of Pedestrian and Evacuation Dynamics, Pedestrian and Evacuation Dynamics, Springer: Cham, pp. 1275-1289.

[15] Moussaïd, M., Helbing, D., and Theraulaz G. (2011), How simple rules determine pedestrian behavior and crowd disasters, Proceedings of the National Academy of Sciences of the United States of America, 108(17), 6884-6888.

[16] Plaue, M., Chen, M.-J., Bärwolff, G., and Schwandt, H. (2011), Trajectory extraction and density analysis of intersecting pedestrian flows from video recordings. In Stilla, U. et al. (eds.), Photogrammetric Image Analysis, ISPRS Conference, PIA 2011, Lecture Notes in Computer Science 6952, Springer: Berlin Heidelberg, ISBN 978-3-642-24392-9, pp. 285296.

[17] Predtechenskii, V.M., and Milinskii, A.I. (1978), Planning for Foot Traffic Flow in Buildings. Amerind Publishing: New Delhi; translation of Proekttirovanie Zhdanii s. Uchetom Organizatsii Dvizheniya Lyuddskikh Potokov (1969), Stroiizdat: Moscow.

[18] Radzihovsky, L., and Clark, N.A. (2003), Comment on "Freezing by heating in a driven mesoscopic system". Phys. Rev. Lett., 90(18), 189603.

[19] Schadschneider, A., Cellular automaton approach to pedestrian dynamics-Theory. In [20], pp. 75-85, ISBN 978-3-540-42690-5.

[20] Schreckenberg, M., and Sharma, S.D. (eds.) (2002), Pedestrian and Evacuation Dynamics, Springer-Verlag Berlin Heidelberg. ISBN 978-3-540-42690-5.

[21] Schadschneider, A. et al. (2009), Evacuation dynamics: Empirical results, modeling and applications, Encyclopedia of Complexity and Systems Science, 3142-3176.

[22] Weidmann, U., Transporttechnik der Fußgänger - transporttechnische Eigenschaften des Fußgängerverkehrs (Literaturstudie), Schriftenreihe der IVT 90 (March 1993) in German. 